\title{
Optimization of a simplified method for fruit phenolic extraction and analysis to be used in olive breeding.
}

\author{
G. Fernández, L. García-Vico, C. Sanz and A.G. Pérez1 \\ Department Biochemistry and Molecular Biology of Plant Products, \\ Instituto de la Grasa, Consejo Superior de Investigaciones Científicas (CSIC), Sevilla, Spain. \\ agracia@cica.es
}

Phenolic compounds are generally accepted as key components of virgin olive oil with a great impact on its organoleptic and health promoting properties. However, olive phenolic components are not commonly used as quality traits in olive breeding programs mainly due to the difficulties of evaluating a large number of new genotypes with very little oil production at the early stages of breeding. In the present work, we describe and optimize a fast and reliable method for the extraction and further analysis of the main phenolic compounds found in olive fruit. The analytical methodology has been validated with the two most relevant olive varieties grown in Spain, "Picual" and "Arbequina", which possess very different phenolic profiles. The significant correlation found between specific phenolic compounds, or groups of phenolic compounds in the olive fruit analyzed with this method, and the phenolic content of virgin olive oil suggest that this method may be a very useful predictive tool which could prevent the selection of olive genotypes whose oils will never reach an optimum phenolic content. breeding.

Keywords: Olea europaea L., olive, fruits, virgin olive oil, phenolic compounds,

\section{INTRODUCTION}

Virgin olive oil (VOO) represents the main lipid source in the Mediterranean diet. It is one of the oldest known plant oils and due to its unique extraction process contains significant amounts of biologically active metabolites (e.g., phenolic compounds, tocopherols, sterols, volatile compounds and pigments) which enhance its nutritional and organoleptic properties (Visioli and Bernardini, 2013). In this sense, it is generally accepted that the phenolic compounds are the VOO components most directly associated with its health related properties (Bernardini and Visioli, 2017). In fact, the scientific evidences on the ability of VOO phenolic components to reduce chronic inflammation and oxidative damage has led the European Union to approve a health claim on olive oil polyphenols which may be applied only for oils containing at least $250 \mathrm{mg} / \mathrm{kg}$ of hydroxytyrosol $\quad(3,4-$ DHPEA) and its derivatives (Commission Regulation (EU) 432/2012). Besides their health promoting properties, VOO phenolic components also have important organoleptic implications given that they are associated to the bitter and pungent sensory notes characterizing VOO (Mateos et al., 2004).

VOO profile comprise a wide range of phenolic compounds belonging to different chemical classes such as secoiridoids, lignans, flavonoids and simple phenolic alcohols and acids. There are many differences in the phenolic profiles among olive cultivars (GarcíaRodríguez et al., 2017; García-González, Tena, and Aparicio, 2010) due to genetic factors 
(Pérez et al., 2014), the agronomic conditions (Romero and Motilva, 2010), and/or the industrial procedures used during the oil extraction (El Riachy et al., 2011). The biosynthesis of the phenolic compounds responsible for the functional and sensory quality of VOO takes place when enzymes and substrates are brought into contact during the milling of olive fruits, the first step in the VOO extraction process. Previous studies have demonstrated that the composition and biochemical status of the olive fruit are the most important variables defining the organoleptic and nutritional quality of the oil. Thus, the phenolic profile of VOO mainly depends on the phenolic content of the olive fruit (GómezRico et al., 2008) and the activity of hydrolytic and oxidative enzymes such as $\beta$-glucosidase, polyphenol oxidase and peroxidase (Romero-Segura et al., 2011). Secoiridoid compounds are the key phenolic components in all olive products. Oleuropein, ligstroside and demethyloleuropein are the main phenolic glycosides found in the olive fruit, and their hydrolytic derivatives, identified as the dialdehydic forms of decarboxymethyloleuropein and ligstroside aglycones (3,4-DHPEA-EDA and $p$-HPEA-EDA, respectively), and the aldehydic forms of oleuropein and ligstroside aglycones (3,4-DHPEA-EA and $p$-HPEA-EA, respectively), are the most abundant phenolic components in most VOOs.

Due to their health promoting and organoleptic properties, phenolic compounds are currently being used as quality markers for VOO. However, phenolic components are not commonly used as quality traits in olive breeding programs, mainly due to the difficulties for evaluating oil phenolic composition in large number of samples and the limited knowledge on the genetic and environmental factors that may influence phenolic composition. In this sense, recent studies have described significant correlations between the composition of olive fruits and oils for components such as fatty acids, sterols, tocopherols or squalene (De la Rosa et al., 2016). In order to predict the phenolic composition of the oil from the analysis of the fruits, it is important to have a reliable and simple analytical methodology to characterize phenolic composition of olive fruits.

Despite the numerous techniques applied and the large amount of published literature on the subject, olive cultivars are far from being fully characterized regarding phenolic compounds (García-Rodríguez et al., 2017). Traditionally, conventional liquidliquid or solid phase extraction methods have been used to extract phenolic compounds from olive fruits and oils. Methanol/water is probably the solvent most used in these protocols usually involving combination of several extracts, and further washing, evaporation and dissolution steps but the use of other solvents have also been reported (Del Río et al., 2003; García et al., 2016). In a similar way, different sample processing techniques have been used in these extraction protocols: fresh olive tissue (Romero et al., 2017), frozen tissue (Gómez-Rico et al., 2008) or freeze-dried samples (Klen et al., 2015).

The aim of this study was to describe a fast and reliable method for the extraction and analysis of olive fruit phenolic compounds which could be applied to a large number of samples, such as those usually associated to olive breeding programs, and that olive breeders could use as a predictive tool.

\section{MATERIAL AND METHODS}

\section{Plant material}

Olive fruits (Olea europaea cv. Picual and Arbequina) used for the optimization of the extraction method were cultivated at the experimental fields of Instituto de la Grasa (Seville, Spain) and collected at an average maturity index of 2.5 (turning stage). Correlations studies among different phenolic compounds were carried out with 25 samples of fruits and oils obtained from Picual and Arbequina trials planted in different olive growing areas of Andalusia. 


\section{Olive oil extraction}

Olive oil was extracted using an Abencor analyzer (Comercial Abengoa, S.A., Seville, Spain) that simulates the industrial process of VOO production on a laboratory scale (Martínez, et al., 1975). Processing parameters have been precisely described in a previous study (Pérez et al., 2014).

\section{Extraction of fruit phenolic compounds}

Two types of samples were used for the extraction of phenolic compounds, fresh pulp tissue and pulp tissue frozen with liquid $\mathrm{N}_{2}$. Frozen samples were stored at $-80^{\circ} \mathrm{C}$ for up to 3 months. Both samples were process in the same way: longitudinal pieces of mesocarp tissue were cut from 20 olive fruits and grinded in dimethyl sulfoxide DMSO ( $6 \mathrm{ml} / \mathrm{g}$ of fruit) or submerged in the same solvent at $4^{\circ} \mathrm{C}$ for different periods of time $(24,48,72 \mathrm{~h}, 1$ week and 1 month). Syringic acid (24 $\mathrm{gg} / \mathrm{ml}$ DMSO) was used as internal standard. All the phenolic extracts obtained were filtered through a $0.45 \mu \mathrm{m}$ mesh nylon and kept at $-20^{\circ} \mathrm{C}$ until HPLC analysis.

\section{Extraction of VOO phenolic compounds}

VOO phenolic compounds were isolated by solid phase extraction (SPE) on a diolbonded phase cartridge (Supelco, Bellefonte, PA) following a previously described procedure (Mateos, et al., 2001).

\section{Analysis of phenolic compounds}

Phenolic extracts from fruits and oils were analyzed by HPLC on a Beckman Coulter liquid chromatography system equipped with a System Gold 168 detector, a solvent module 126, an auto sampler module 508 and a Waters column heater module following a previously described methodology (García-Rodríguez et al., 2011). A Superspher RP 18 column ( $4.6 \mathrm{~mm}$ i.d. x $250 \mathrm{~mm}$, particle size $4 \mu \mathrm{m}$ : Dr Maisch GmbH, Germany) at flow rate

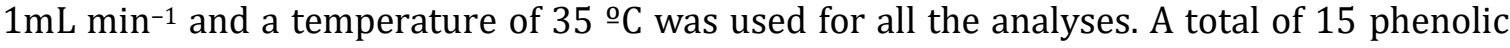
compounds were analyzed in fruit phenolic extracts: hydroxytyrosol-4-glucoside, hydroxytyrosol-1-glucoside, demethyloleuropein, verbascoside, luteolin-7-glucoside, demethylligstroside, rutin, oleuropein, comselogoside, ligstroside, luteolin 3,4-DHPEA-EA, apigenin and $p$-HPEA-EA. The last four compounds were also analyzed in VOO extracts in which 12 other phenolic compounds were also detected: hydroxytyrosol, tyrosol, vanillic acid, vainillin, p-coumaric acid, hydroxytyrosol acetate, 3,4-DHPEA-DEA, $p$-HPEA-DEA, pinoresinol, acetoxypinoresinol, and ferulic acid. The tentative identification of compounds by their UV-vis spectra was confirmed by HPLC/ESI-qTOF-HRMS. (García-Rodríguez et al., 2017).

\section{Statistical analysis}

Data were statistically evaluated using STATISTICA (Statsoft Inc., Tulsa, OK, USA). Correlations among phenols or group of phenols were analyzed using Pearson's correlations. ANOVA and separation of the means was obtained at $p \leq 0.05$ by least significance differences (LSD).

\section{RESULTS AND DISCUSSION}

The main goal of this study was to optimize a simple and reliable method to be used for phenolic profiling in a large set of olive samples. DMSO is a dipolar aprotic solvent widely used in pharmacology for its ability to penetrate biological membranes. Due to its activity as free radical scavenger, very useful to avoid oxidation of plant extracts, DMSO has been previously used in the extraction of plant phenolic compounds (Del Río et al., 2003). Table 1 and 2 show the phenolic composition of olive extracts obtained by homogenizing olive pulp 
tissue in DMSO or by simple immersion of longitudinal pieces of pulp tissue in DMSO for different periods of time. The content of the main phenolic glycosides analyzed in Picual and Arbequina fruits is consistent with previous data on the phenolic profile of both cultivars (Talhaoui et al, 2016; Romero et al., 2017). Although significantly higher contents of some phenolic compounds were found in the extracts obtained by homogenization of the olive tissue nonsignificant differences were found in the total phenolic content of Arbequina extracts obtained by simple immersion in DMSO for more than 48h (Table1). Despite the significant differences found among Picual extracts (Table 2) it is important to point out that total phenolic content in $48 \mathrm{~h}$ extracts is only $5 \%$ lower than that found in those extracts obtained from homogenized tissue. It is also remarkable that hydroxytyrosol and tyrosol glucosides contents were generally lower in those extracts. Data presented in Table 1 and 2 suggest that simple immersion of pulp tissue in DMSO for more that $48 \mathrm{~h}-72 \mathrm{~h}$ could be a reliable and fast method for the extraction of olive phenolic compounds. The simplicity of the method, with very little manipulation of the samples and very stable extracts could be a very useful tool to analyze large number of samples. In fact, in order to process a high number of samples, many experimental protocols for phenolic analysis freeze tissue samples with liquid nitrogen and kept them at $-80^{\circ} \mathrm{C}$ until extraction and analysis. In this sense, it is important to emphasize that freezing of the olive pulp and the subsequent thawing of the tissue causes a significant alteration of the phenolic composition. In a recent study (García-Vico et al. 2017), we have described that olive freezing produces a clear reduction in the phenolic content, with a very important decrease of oleuropein and demethyloleuropein levels and a minor reduction in the content of verbascoside and other olive phenolic glycosides. Although, it is clear that hydrolytic and oxidative reactions could contribute to this phenolic degradation, the alteration induced by freezing seems to be more related to the release of $\beta$-glucosidase proteins due to intracellular damage. This enzyme, with very high substrate and product specificity, exhibits maximal specificity towards oleuropein, followed by demethyloleuropein, but is not active on verbascoside (RomeroSegura et al., 2011). Figure 1 shows the differences found in the phenolic profiles of fresh and frozen Arbequina and Picual fruits extracted by immersion in DMSO (72h). Oleuropein, the main phenolic glucoside, reduces its content in frozen olive tissues around $60 \%$ in both olive cultivars. The degradation of oleuropein and demethyloleuropein seems to be associated to the freezing process and is independent of the subsequent storage time at $80^{\circ} \mathrm{C}$ (data not shown). These data highlight the need of using fresh tissue for fruit phenolic profiling.

In order to evaluate the feasibility of the optimized methodology to be used as a predictive tool in olive breeding 25 samples of olives fruits and their corresponding oils were analyzed. Samples were harvested at different ripening stages from different Picual and Arbequina orchards in Southern Spain. Oils were obtained using the Abencor system under standard conditions, and VOO phenolic compounds extracted and analyzed as described in Material and Methods Section. Pearson's correlation coefficients were computed using the data obtained from all the fruits and oils analyzed. Table 3 shows the correlation coefficients found among total phenolic content, total secoiridoid compounds and the main classes of phenolic components found in fruits and oils: oleuropein derivatives (oleuropein and demethyloleuropein), ligstroside derivatives (ligstroside and demthylligstroside), hydroxytyrosol derivatives (3,4-DHPEA-EA, 3,4-DHPEA-EDA, 3,4-DHPEA-Acetate), tyrosol derivatives (p-HPEA-EA, p-HPEA-EDA) and lignans (acetoxypinoresinol and pinoresinol). 
Table 3. Pearson correlation coefficients among the main groups of phenolic compounds analyzed in fruits and oils from 25 Picual and Arbequina orchards.

Marked correlations are significant at: ${ }^{*} \mathrm{p}<0.05$

A significant positive correlation was found between total fruit phenolics and total VOO phenolic content $(r=0.758)$ and even a slightly higher coefficient was calculated for fruit secoiridoids compounds and the total phenolic content of the VOO $(r=0.786)$. A very high correlation was also found between oleuropein derivatives present in the olive fruit and the hydroxytyrosol derivatives present in the VOO $(r=0.754)$. On the contrary, a negative correlation was found between ligstroside derivatives in the fruit and tyrosol containing compounds in the oil $(\mathrm{r}=-0.246)$.

\section{CONCLUSION}

The experimental data reported in this paper suggest that the analytical procedure

\begin{tabular}{c|lcccc}
\hline \multicolumn{5}{c}{} & \multicolumn{5}{c}{ Olive fruit } \\
\cline { 3 - 6 } & & $\begin{array}{c}\text { Total } \\
\text { phenols }\end{array}$ & $\begin{array}{c}\text { Total } \\
\text { Secoiridoids }\end{array}$ & $\begin{array}{c}\text { Oleuropein } \\
\text { derivatives }\end{array}$ & $\begin{array}{c}\text { Ligstroside } \\
\text { derivatives }\end{array}$ \\
\cline { 2 - 6 } & Total phenols & $* 0.758$ & $* 0.786$ & $* 0.795$ & 0.361 \\
$\Xi$ & Total Secoiridoids & $* 0.757$ & $* 0.785$ & $* 0.795$ & 0.352 \\
$\overline{0}$ & Hydroxytyrosol der. & $* 0.744$ & $* 0.746$ & $* 0.754$ & $* 0.532$ \\
& Tyrosol der. & 0.359 & $* 0.422$ & $* 0.430$ & -0.246 \\
& Lignans & $* 0.582$ & $* 0.590$ & $* 0.585$ & $* 0.388$ \\
\hline
\end{tabular}

described here for olive phenolic extraction (immersion of longitudinal fruit tissue pieces in DMSO for $72 \mathrm{~h}$ at $4^{\circ} \mathrm{C}$ ) is a simple and reliable procedure for olive phenolic profiling. The excellent correlations found between the phenolic profiles of fruits and oils indicate that this methodology could be used as a predictive tool in olive breeding. Taking into account the well-known influence of the phenolic fraction in the sensorial and health-promoting properties of VOO, the analysis of the fruit phenolic profile can be very useful to identify olive cultivars producing oils with an improved nutritional quality.

\section{ACNOWLEDGEMENTS}

This work was funded by Programa Nacional de Recursos y Tecnologías agroalimentarias financed by the Spanish Government, project AGL2015-67652.

\section{Literature cited}

Bernardini, E., and Visioli, F. (2017). High quality, good health: the case for olive oil. Eur. J. Lipid Sci. Technol. 119:1500505. doi: 10.1002/ejlt.201500505

De la Rosa R., Arias Calderón, R., Velasco, L, and León L. (2016). Early selection for oil quality components in olive breeding progenies. Eur. J. Lipid. Sci. Technol. 118, 1160-1167

Del Río J.A., Baidez, A.G., Botía, J.M. (2003). Enhancement of phenolic compounds in olive plants (Olea europaea L.) and theirs influence on resistance against Phytoctora sp. Food Cehm. 83:75-78. 
El Riachy, M., Priego-Capote, F., León, L., Rallo, L., Luque de Castro, M. D. (2011). Hydrophilic antioxidants of virgin olive oil. Part 2: Biosynthesis and biotransformation of phenolic compounds in virgin olive oil as affected by agronomic and processing factors. Eur. J. Lipid Sci. Technol, . 113, 692-707.

European Comunities (2012). Commision Regulation (EU) No 432/2012 establishing a list of permitted health claims made on foods, other than those referring to the reduction of disease risk and to children's development and health. Off. J. Eur. Union, L 136/1-40.

García-González, D.L., Tena, N. \& Aparicio, R. (2010). Quality characterization of the new virgin olive oil var. Sikitita by phenols and volatile compounds. J. Agric. Food Chem., 58, 8357-8364.

García-Rodríguez, R., Belaj, A., Romero-Segura, C., Sanz, C., and Pérez, A. (2017). Exploration of genetic resources to improve the functional quality of virgin olive oil. J. Funct. Foods 38, 1-8.

García-Rodríguez, R., Romero-Segura, C., Sanz, C., Sánchez-Ortiz, A., and Pérez, A. (2011). Role of polyphenol oxidase and peroxidase in shaping the phenolic profile of virgin olive oil. Food Res. Int. 44, 265-635.

García, A., Rodríguez-Juan, E., Garcia-Rodríguez, G, Rios, J.J., Fernandez, J. (2016). Extraction of pehnolic compounds from virgin olive oil by Deep eutectic solvents. Food Chem. 197: 554-561.

García-Vico, L., García-Rodríguez, R., Sanz, C., Pérez, A.G. (2017). Biochemical aspects of olive freezing-damage: Impact on the phenolic and volatile profiles of virgin olive oil. LWT - Food Science and Technology 86: 240-246

Gómez-Rico, A., Fregapane, G., and Salvador, M. D. (2008). Effect of cultivar and ripening on minor components in Spanish olive fruits and their corresponding virgin olive oils. Food Res. Int. 41, 433-440. doi: 10.1016/j.foodres.2008.02.003.

Klen, T., Wondra, A.G., Vrhovšek, U., Sivilotti, P., Mozetic Vodopivec, B. (2015). Olive fruit phenols transfer, transformation, and partition trail during laboratory-scale olive oil processing. J. Agric. Food Chem. 63, 45704579.

Martínez, J. M., Muñoz, E., Alba, J., \& Lanzón, A. (1975). Report about the use of the 'Abencor' analyser. Grasas y Aceites, 26, 379-385.

Mateos, R., Cert, A., Pérez-Camino, M.C., and García, J.M. (2004). Evaluation of virgin olive oil bitterness by quantification of secoiridoid derivatives. J. Am. Oil. Chem. Soc. 81, 71-75.

Mateos, R., Espartero, J.L., Trujillo, M., Rios, J.J., Leon Camacho, M., Alcudia, F., Cert, A. (2001) Determination of phenols, flavones, and lignans in virgin olive oils by solid-phase extraction and high-performance liquid chromatography with diode array ultraviolet detection. J. Agric. Food Chem., 49, 2185-2192.

Pérez, A.G., León, L., Pascual, M., Romero-Segura, C., Sánchez-Ortiz, A., de la Rosa, R.\& Sanz, C. (2014). Variability of virgin olive oil phenolic compounds in a segregating progeny from a single cross in Olea europaea L. and sensory and nutritional quality implications. PLOS ONE 9, e92898

Romero, C, Medina, E, Mateo, M.A. and Brenes, M. (2017). Quantification of bioactive compounds in 'Picual' and 'Arbequina' olive leaves and fruits. J Sci Food Agric; 97, 1725-1732

Romero, M.P. and Motilva, M.J. (2010) Effect of climatic conditions on quality of virgin olive oil. In Olives and Olive Oil in Health and Disease Prevention; Preedy, V.R., Watson, R.R., Eds.; Elsevier Inc: San Diego (CA), pp. 43-50.

Romero-Segura, C., García-Rodríguez, R., Sanz, C., and Pérez, A. G. (2011). Virgin olive phenolic profile as a result of the anabolic and catabolic enzymes status in the olive fruit. Acta Hort. 924, 379-384. doi: 10.17660/ActaHortic.2011.924.47

Talhaoui N., Gómez-Caravaca, A. M., León, L., De la Rosa, R., Fernández-Gutiérrez, A., Segura-Carretero, A. (2016) From Olive Fruits to Olive Oil: Phenolic compound Transfer in Six Different Olive Cultivars Grown under the Same Agronomical Conditions Int. J. Mol. Sci. 17, 337; doi:10.3390/ijms17030337 www.mdpi

Visioli, F., and Bernardini, E. (2011). Extra virgin olive oil's polyphenols: Biological activities. Curr. Pharm. Design. 17, 786-804. 
Figure captions

Figure 1: Content of the main phenolic glycosides (demethyloleuropein, oleuropein, ligstroside, verbascoside and luteolin-7-glucoside) found in fresh and frozen pulp of Arbequina (A) and Picual (B) olive fruits. 
Table 1. Main phenolic compounds analyzed in Arbequina fruits by means of different extraction methods using DMSO.

Main phenolic compounds in Arbequina fruits $(\mu \mathrm{g} / \mathrm{g})$

\begin{tabular}{|c|c|c|c|c|c|c|c|c|}
\hline & Hty-G & Tyr-G & DemO & Oleuropein & Ligstroside & Verbascoside & Luteolin-G & Total \\
\hline \multicolumn{9}{|c|}{ Extraction method } \\
\hline Grinded tissue & $645.7 a^{*}$ & $60.1 b$ & $7736.0 \mathrm{~b}$ & $21314.1 \mathrm{c}$ & $1014.0 \mathrm{a}$ & $3028.1 b$ & $1155.4 b$ & $31978.5 b$ \\
\hline $24 \mathrm{~h}$ & $704.6 b$ & $51.4 \mathrm{a}$ & $7028.9 a$ & $15765.5 a$ & $1090.5 a$ & $2700.6 a$ & $916.4 \mathrm{a}$ & 28257.9a \\
\hline $48 \mathrm{~h}$ & $739.5 c$ & $53.3 a$ & $7766.0 \mathrm{~b}$ & 16899.0ab & $1162.2 \mathrm{~b}$ & $2920.6 b$ & $975.9 \mathrm{ab}$ & $30516.6 b$ \\
\hline $72 \mathrm{~h}$ & $758.4 c$ & $54.4 \mathrm{ab}$ & $7930.0 \mathrm{~b}$ & 17048.3ab & $1197.4 b$ & $2940.5 b$ & 1039.6ab & $30700.1 b$ \\
\hline 1 week & $749.3 c$ & $54.5 \mathrm{ab}$ & $7939.2 b$ & 16818.5ab & $1196.5 b$ & 2911.0b & 1030.3ab & $30699.4 b$ \\
\hline 1 month & $817.1 \mathrm{~d}$ & $47.9 \mathrm{a}$ & $8089.2 \mathrm{c}$ & $17704.8 \mathrm{~b}$ & $1258.5 c$ & $3023.2 \mathrm{~b}$ & 1051.3ab & $31992.9 \mathrm{~b}$ \\
\hline
\end{tabular}

* Values represent the mean of three independent analyses. Different letters in the same column indicate significant differences (p $\leq 0.05$ )

**Hty-G (hydroxytyrosol-4-glucoside); Tyr-G (Tyrosol-glucoside); DemO (demethyloleuropein); Luteolin-G (luteolin-7-glucoside)

Table 2. Main phenolic compounds analyzed in Picual fruits by means of different extraction methods using DMSO.

Main phenolic compounds in Picual fruits $(\mu \mathrm{g} / \mathrm{g})$

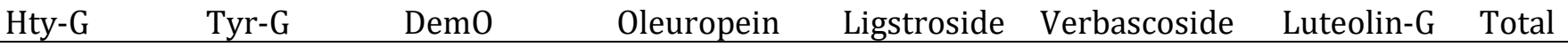

Extraction method

\begin{tabular}{|c|c|c|c|c|c|c|c|c|}
\hline Grinded tissue & $353.1 \mathrm{a}$ & $30,2 a$ & $450.5 \mathrm{ab}$ & $32197,5 d$ & $3359,5 c$ & $3562.3 \mathrm{~b}$ & $918.7 b$ & $40872.3 d$ \\
\hline $24 \mathrm{~h}$ & $492.9 b$ & $45.0 \mathrm{~b}$ & $428.9 a$ & $29408.7 a$ & $3149.9 a$ & $3351.8 \mathrm{a}$ & $739.2 a$ & $37616.2 \mathrm{a}$ \\
\hline $48 \mathrm{~h}$ & $509.6 \mathrm{bc}$ & $52.0 c$ & 456.5abc & $30422.5 a b c$ & $3232.1 b$ & 3429.6ab & $768.6 a$ & 38871.0abc \\
\hline $72 \mathrm{~h}$ & $512.1 c$ & $52.8 c$ & $466.8 \mathrm{bc}$ & $30666.6 b c$ & $3248.5 b$ & 3488.1ab & $794.2 \mathrm{ab}$ & $39229.2 b c$ \\
\hline 1 week & $505.3 \mathrm{bc}$ & $53.1 c$ & $460.3 \mathrm{ab}$ & 29820.8ab & 3208.1ab & $3349.1 \mathrm{a}$ & 767.1a & 38163.7ab \\
\hline 1 month & $551.0 \mathrm{~d}$ & $57.2 \mathrm{~d}$ & $497.9 c$ & $30985.9 c$ & $3402.5 c$ & 3426.3ab & $781.4 \mathrm{a}$ & $39702.2 \mathrm{~cd}$ \\
\hline
\end{tabular}

* Values represent the mean of three independent analyses. Different letters in the same column indicate significant differences (p $\leq 0.05)$

**Hty-G (hydroxytyrosol-4-glucoside); Tyr-G (Tyrosol-glucoside); Dem0 (demethyloleuropein); Luteolin-G (luteolin-7-glucoside) 
Figure 1

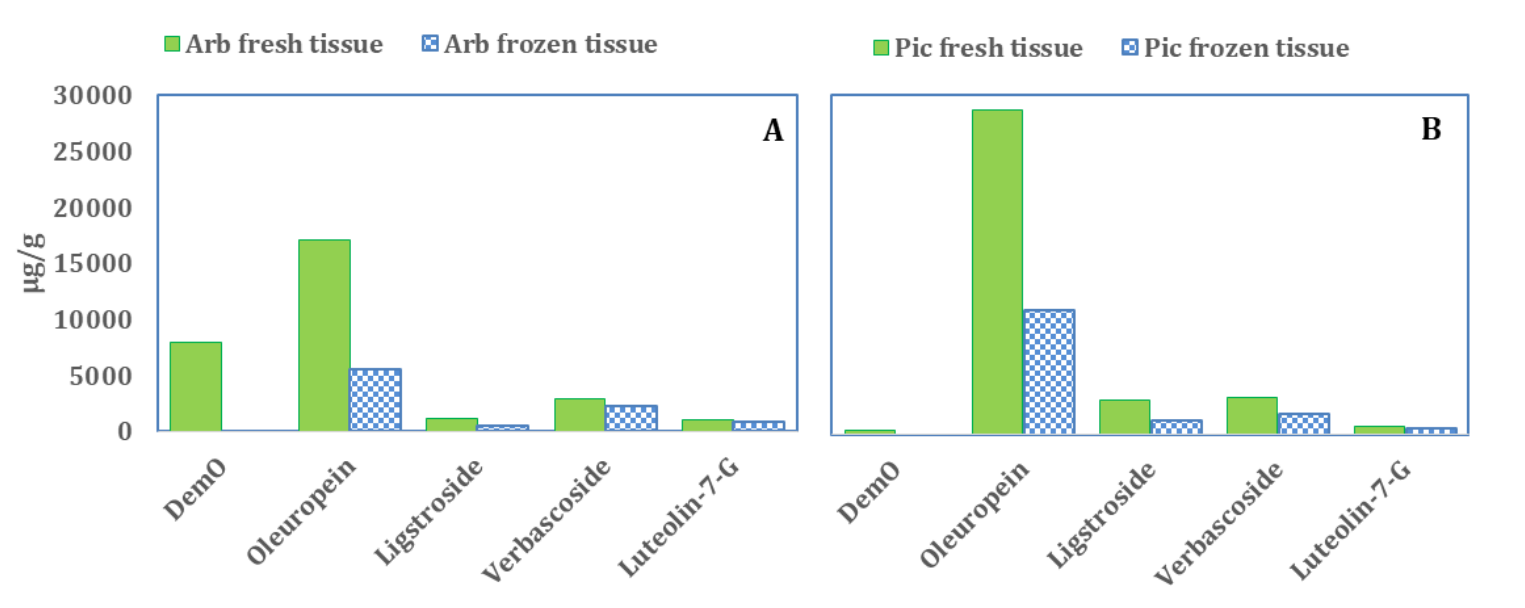

